\title{
Facile One-Pot Ultrasound-Assisted Reduction Synthesis of Pd/C Nanocatalyst for Green Suzuki-Miyaura Coupling
}

\author{
Jiazhe $\mathrm{Li}^{*}$ and Xuefeng Bai* \\ College of Materials Science and Chemical Engineering, \\ Harbin Engineering University \\ Harbin 150001, China \\ sweet_li@126.com
}

\author{
Xuefeng Bai* \\ Institute of Petrochemistry \\ Heilongjiang Academy of Sciences \\ Harbin 150040, China \\ tommybai@126.com
}

\begin{abstract}
Palladium nanoparticles (Pd NPs) supported on active carbon was prepared via ultrasonically treating $\mathbf{P d}$ precursors in ethylene glycol without any protective agents. The $\mathrm{Pd} / \mathrm{C}$ nanocatalysts were characterized using $\mathrm{X}$-ray diffraction (XRD), scanning electron microscopy (SEM), transmission electron microscopy (TEM), N2-adsorption analysis, and X-ray photoelectron spectrometry (XPS). The results suggested that Pd NPs with an average particle size of $7.54 \mathrm{~nm}$ are formed in $\mathrm{Pd} / \mathrm{C}-600 \mathrm{~W}$. Pd NPs of Pd/C-600 W nanocatalysts were highly distributed on the surface of active carbon. Even under mild conditions, Pd/C-600 W catalyst exhibited a better catalytic performance than that of $\mathrm{Pd} / \mathrm{C}$-T catalysts in Suzuki-Miyaura coupling reactions. The yield of 4-bromonitrobenzene reached 94.28\% with only $0.1 \mathrm{mmol} \% \mathrm{Pd} / \mathrm{C}-600 \mathrm{~W}$ catalyst. Pd/C-600 W catalyst could be easily separated by filtration. It could be recycled four times with limited activity decrease. The catalytic activity and recyclability of $\mathrm{Pd} / \mathrm{C}-600 \mathrm{~W}$ is higher than $\mathrm{Pd} / \mathrm{C}-\mathrm{T}$. This ascribed to smaller size and more uniform dispersion of Pd/C-600 W.
\end{abstract}

Keywords-ultrasonic reduction, pd/c nanocatalyst, heterogeneous catalysts, green suzuki-miyaura coupling reaction.

\section{INTRODUCTION}

Palladium (Pd)-based nanometerials have get great attention as catalyst due to its high activity in various catalysis systems including the well-known Suzuki [1, 2], Sonogashira and Heck, which are the vital way to construct carbon-carbon bond.

To achieve the high activity of the Pd nanocatalyst, controlling the size and increasing the active sites are important. A large amount of attempts have been made to synthesize Pd NPs using complicated procedures, such as adding protective agents to prevent Pd NPs from reuniting. However, these methods lead to the reduction of active sites resulted from absorbing on the surface of nanocatalyst by protective agents.

Various approaches have been used to prepared $\mathrm{Pd}$ nanocatalsyst, such as impregnation, chemical reduction [3], electrochemical deposition [4], microwave assisted chemical reduction and ultrasonic reduction. Among various methods for preparing nanocatalysts, the ultrasonic reduction is more promising because of its facile process, low cost, mild reaction conditions and potential for large-scale production. The mechanism of ultrasonic reduction arise from acoustic cavitation, which is the formation, growth and implosive collapse of bubbles in liquid. The extreme transient conditions introduced by ultrasound produced hot spots with temperature up $5000 \mathrm{~K}$, pressure above 1000 atmospheres, heating and colling rates exceeding $1010 \mathrm{~K} \cdot \mathrm{s}^{-1}$, permitting the preparation of catalysts in mild conditions instead of high temperature and strong reductants [5].

Therefore, in this research, the supported $\mathrm{Pd} / \mathrm{C}$ nanocatalyst is synthesized from $\mathrm{Na}_{2} \mathrm{PdCl}_{4}$ as $\mathrm{Pd}$ precursor by one-pot ultrasonic reduction, employed ethylene glycol as reductant and solvent without any protective agent in reaction system. The catalytic properties and recyclability of $\mathrm{Pd} / \mathrm{C}-600 \mathrm{~W}$ for the Suzuki-Miyaura coupling reaction of 4-bromotoluene and phenylboronic acid were examined.

\section{Preparation of PD/C Nanocatalyst}

Firstly, a commercially available coconut shell activated carbon was crushed and sieved. In a typical ultrasonic synthesis, $0.5 \mathrm{~g}$ active carbon (200-300 mesh) was dispersed in $30 \mathrm{~mL}$ of ethylene glycol at an ultrasonic power of $100 \mathrm{~W}$ for $30 \mathrm{~min}$. Then, a required amount $(5 \mathrm{wt} \% \mathrm{Pd})$ of the precursor solution $\left(\mathrm{Na}_{2} \mathrm{PdCl}_{4}, 0.5 \mathrm{~mol} / \mathrm{L}\right)$ was sonicated at ultrasonic power of $600 \mathrm{~W}$ for another $30 \mathrm{~min}$. The final black solid was centrifuged and washed, dried at $40{ }^{\circ} \mathrm{C}$ in an oven for $12 \mathrm{~h}$, and ground into a fine powder. During the reaction process, the reaction temperature was kept at about $30^{\circ} \mathrm{C}$ using the water surrounding the reactor. The obtained catalysts were denoted as "Pd/C-600 W". As a comparison, $\mathrm{Pd} / \mathrm{C}$ nanocatalyst was prepared by conventional water-bath and magnetic stirring without ultrasound, denoted as "Pd/C-T". 


\section{GENERAL PROCEDURES OF SUZUKI COUPLING REACTION}

The required amount of the $\mathrm{Pd} / \mathrm{C}$ nanocatalysts $(0.1$ mmol $\%, 0.25 \mu \mathrm{mol})$ was added to the stirring mixture of 4-bromotoluene (2.5 mmol), $\mathrm{K}_{2} \mathrm{CO}_{3}(691.0 \mathrm{mg}, 5.0 \mathrm{mmol}), 30$ $\mathrm{mL}$ of EtOH/ $\mathrm{H}_{2} \mathrm{O}(1: 1)$ and phenylboronic acid $(365.8 \mathrm{mg}, 3.0$ mmol) followed. The mixture was then stirred at $60^{\circ} \mathrm{C}$ for 60 min. in a water bath and extracted with ethyl acetate $(3 \times 10$ $\mathrm{mL})$. The organic layer was combined and then analyzed by HPLC. In order to check the recycling and stability of $\mathrm{Pd} / \mathrm{C}$ nanocatalyst, $\mathrm{Pd} / \mathrm{C}$ nanocatalyst was collected by centrifugation and then washed thoroughly with $\mathrm{NaOH}$ to remove the redundant phenylboronic acid, followed by water and ethanol to remove the impurity present in the used catalysts.

\section{RESULTS AND DISCUSSIONS}

Fig. 1 showed the XRD patterns of the activated carbon, $\mathrm{Pd} / \mathrm{C}-600 \mathrm{~W}$, and Pd/C-T. Two broad peaks at about $24.6^{\circ}$ and $43.8^{\circ}$ appeared at all samples are assigned to the planes typical for graphite carbons. In the patterns of Fig. 1a and b, the peaks appearing at $40.1^{\circ}, 46.6^{\circ}$ and $68.1^{\circ}$ were due to the presence of face-centered cubic Pd planes (111), (200), (220) [6]. This suggests that Pd was successfully loaded on the surface of active carbon through two different reduction methods. The peak intensity of $\mathrm{Pd} / \mathrm{C}-\mathrm{T}$ nanocatalyst was significantly sharper in shape and higher in intensity. This indicates that $\mathrm{Pd} / \mathrm{C}-600$ W nanocatalyst prepared by ultrasonic method had smaller size.

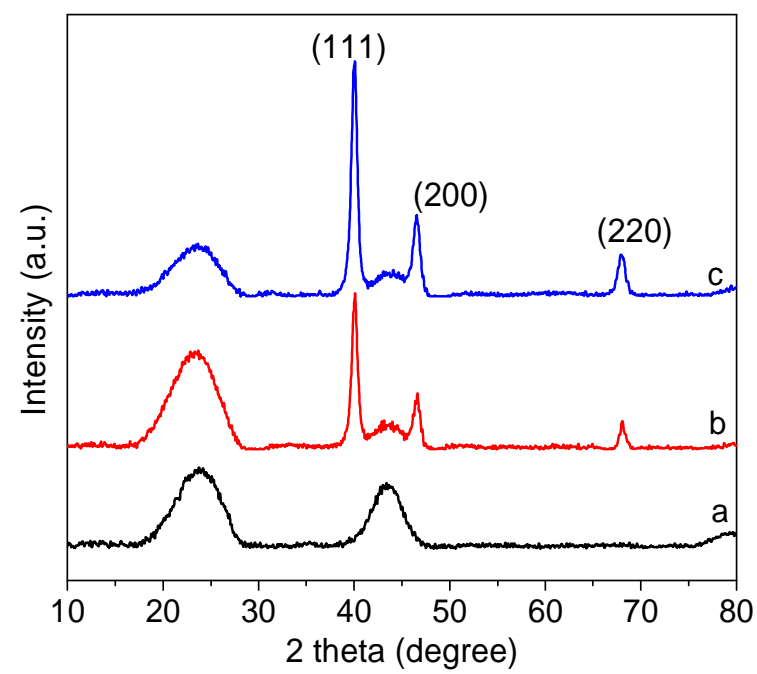

Fig. 1. XRD patterns of (a) activated carbon, (b) Pd/C-600 W and (c) Pd/C-T

The $\mathrm{N}_{2}$ adsorption-desorption isotherms and the corresponding pore size distribution of activated carbon, $\mathrm{Pd} / \mathrm{C}-\mathrm{T}$ and $\mathrm{Pd} / \mathrm{C}-600 \mathrm{~W}$ are depicted in Fig. 2. All the samples present a type I isotherm according to the IUPAC classification and exhibited a $\mathrm{H} 4$ hysteresis loop indicating the typical micropore structure [7]. The overall form of the $\mathrm{N}_{2}$ adsorption-desorption isotherms of $\mathrm{Pd} / \mathrm{C}$ nanocatalysts were similar to that of active carbon, suggesting that they retained ordered structure.
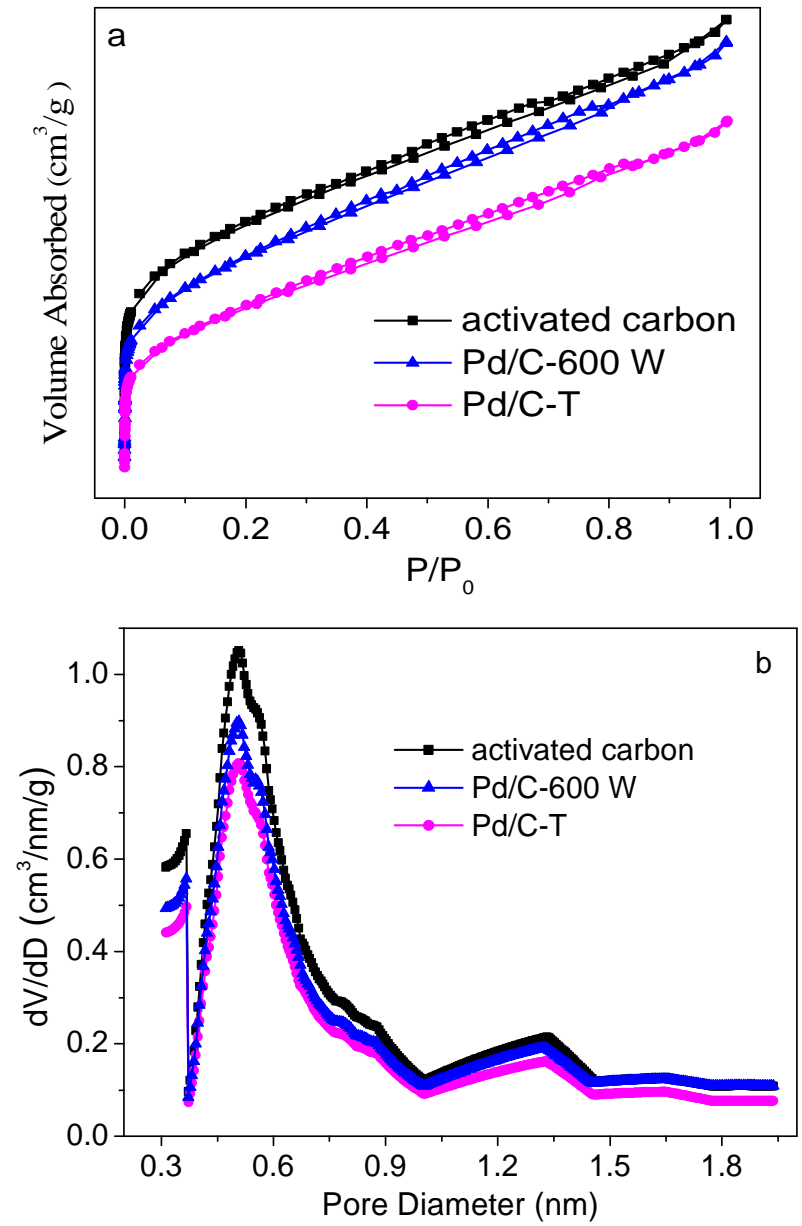

Fig. 2. (a) N2 adsorption-desorption isotherms and (b) Pore size distributions curves of activated carbon, $\mathrm{Pd} / \mathrm{C}-\mathrm{T}$ and $\mathrm{Pd} / \mathrm{C}-600 \mathrm{~W}$

The main textural properties of the samples are shown in Table 1. Compared with the support active carbon, BET surfaces and pore volume of $\mathrm{Pd} / \mathrm{C}-600 \mathrm{~W}$ and $\mathrm{Pd} / \mathrm{C}-\mathrm{T}$ nanocatalysts are decreased. The decrease suggests that $\mathrm{Pd}$ at least partially located inside the channels of support. The reduction of BET surfaces and pore volume are more remarkable for $\mathrm{Pd} / \mathrm{C}-600 \mathrm{~W}$, which may be due to the Pd NPs of $\mathrm{Pd} / \mathrm{C}-\mathrm{T}$ prepared by conventional method have increased size resulted in moving from the channel to surface or edge of support. This result will be evidenced by TEM. According to the adsorption branches using the HK method, the pore distribution of active carbon reveals uniform micropores distribution with pore diameter $0.508 \mathrm{~nm}$, with no significant differences for $\mathrm{Pd} / \mathrm{C}$ catalysts. This demonstrates that the pore structure of active carbon is not destroyed.

TABle 1. TeXtural PROPERTIES OF ACTIVATED CARBon, PD/C-600 W AND PD/C-T.

\begin{tabular}{cccc}
\hline Samples & $\mathrm{S}_{\mathrm{BET}}\left[\mathrm{m}^{2} / \mathrm{g}\right]$ & $\mathrm{D}_{\mathrm{HK}}[\mathrm{nm}]$ & $\mathrm{V}_{\mathrm{HK}}\left[\mathrm{cm}^{3} / \mathrm{g}\right]$ \\
\hline Activated carbon & 1200.0 & 0.508 & 0.9208 \\
Pd/C-600 W & 910.7 & 0.502 & 0.6774 \\
Pd/C-T & 1048.7 & 0.508 & 0.8770 \\
\hline
\end{tabular}


The TEM images and the particle size distribution of $\mathrm{Pd} / \mathrm{C}-600 \mathrm{~W}$ are displayed in Fig. 3. After introduction of Pd, uniform Pd distribution can be observed. The high-resolution transmission electron microscopy (HRTEM) image further demonstrated the lattice fringe was $0.223 \mathrm{~nm}$, which was attributed to the lattice spacing of the Pd (111) plane [8] (Fig. $3 c)$. The Pd NPs size of Pd/C-600W catalyst ranges from 3.77 $\mathrm{nm}-17.87 \mathrm{~nm}$ and the mean size is about $7.54 \mathrm{~nm}$ (Fig. 3e). For the $\mathrm{Pd} / \mathrm{C}-\mathrm{T}$ catalyst, the $\mathrm{Pd}$ NPs tended to aggregate remarkably. This further validates that the ultrasound provides the sufficient volatility and diffusion leading to the formation of highly disperse Pd NPs.

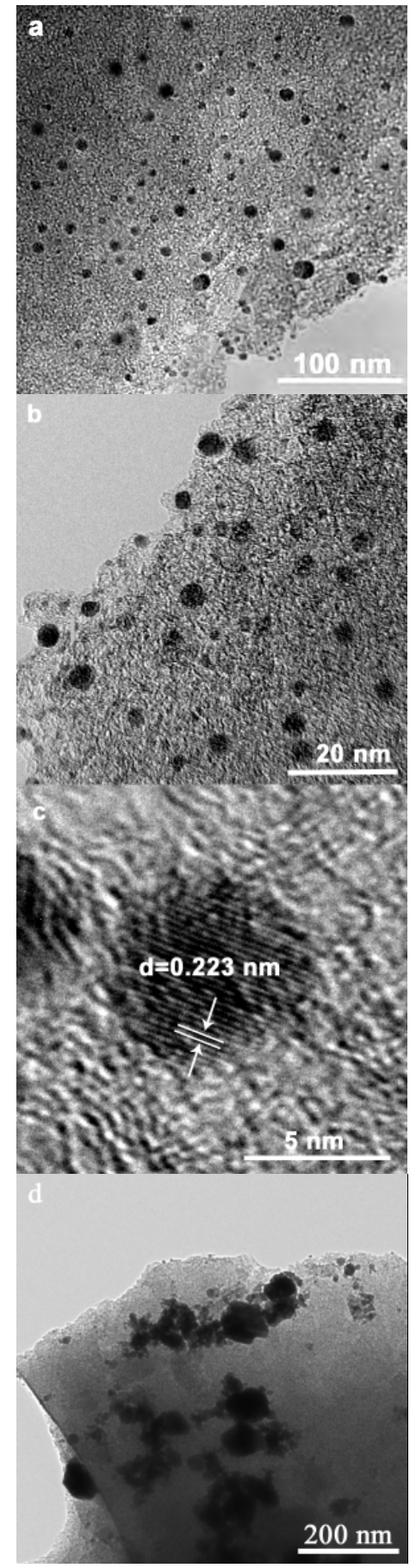

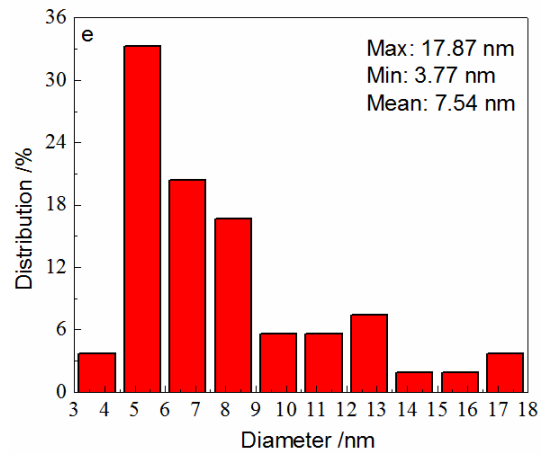

Fig. 3. (a, b) TEM images and (c) HRTEM image of Pd/C-600 W, (d) TEM image of $\mathrm{Pd} / \mathrm{C}-\mathrm{T}$ and (e) particle size distribution of $\mathrm{Pd} / \mathrm{C}-600 \mathrm{~W}$.

The XPS spectrum of the $\mathrm{Pd} 3 \mathrm{~d}$ region consists of four peaks (Fig. 4). The peaks at lower binding energy (335.05 and $340.45 \mathrm{eV}$ ) belong to $\mathrm{Pd}^{0}$, and the other peaks at 337.1 and $342.5 \mathrm{eV}$ are assigned to $\mathrm{Pd}^{2+}$ [8]. The presence of $\mathrm{Pd}^{2+}$ may arise from an incomplete reduction of $\mathrm{Pd}^{2+}$ ions and the oxidation of naked metal Pd atoms under ambient conditions. According to the peak area of zerovalent palladium and bivalent palladium, the similar reduction degrees are determined, about $68 \%$ and $65 \%$ for Pd/C-600W and Pd/C-T.
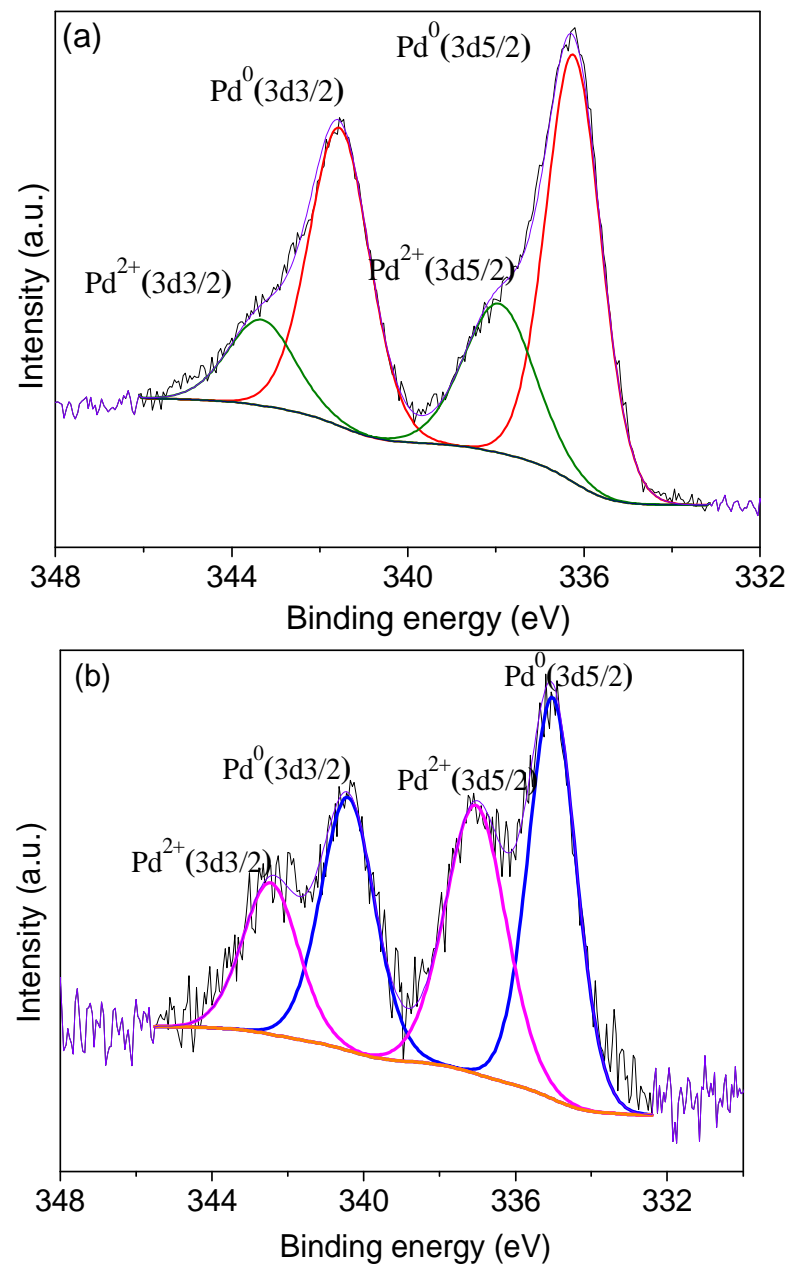

Fig. 4. Pd 3d XPS spectrum: (a) Pd/C-600 W and (b) Pd/C-T 


\section{SUZUKI-MIYAURA COUPLING REACTION}

As shown in Fig. 5a, the yield increase gradually along with the increase of reaction time in catalytic processes of two kinds of catalysts, and the catalytic activity of $\mathrm{Pd} / \mathrm{C}-600 \mathrm{~W}$ prepared by ultrasonic reduction method is superior to the traditional preparation of $\mathrm{Pd} / \mathrm{C}-\mathrm{T}$ catalyst. The conversion of 4-bromotoluene reached to $94.28 \%$ at $60 \mathrm{~min}$, up to $96.09 \%$ at 90 min with only $0.1 \mathrm{mmol} \%$ catalyst. Combined with TEM analysis, it indicates that the dispersion of Pd NPs on the surface of support plays a decisive role to the catalytic activity of catalysts. The high dispersion of Pd NPs is advantageous to expose more active sites and contact more sufficiently with reactants, resulted in increasing the catalytic activity. And the preparation of supported Pd NPs by ultrasonic method in the absence of protective agent can effectively improve its dispersion, obtaining the high catalytic efficiency. Pd/C-600 W nanocatalyst was tested in terms of durability of five cyclic run. Fig. 5b shows that Pd/C-600 W nanocatalyst still exhibits over $90 \%$ after three cycles, and retains $80 \%$ in the fifth cycle. As for the $\mathrm{Pd} / \mathrm{C}-\mathrm{T}$ catalyst, the activity decreased to $50 \%$ after using three cycles. It can be explained that the Pd NPs in the case of $\mathrm{Pd} / \mathrm{C}-600 \mathrm{~W}$ has smaller size and more uniform dispersion, determining more strong interaction between Pd species and support, and further obtained the better recyclability and stability for Pd/C-600W than Pd/C-T.
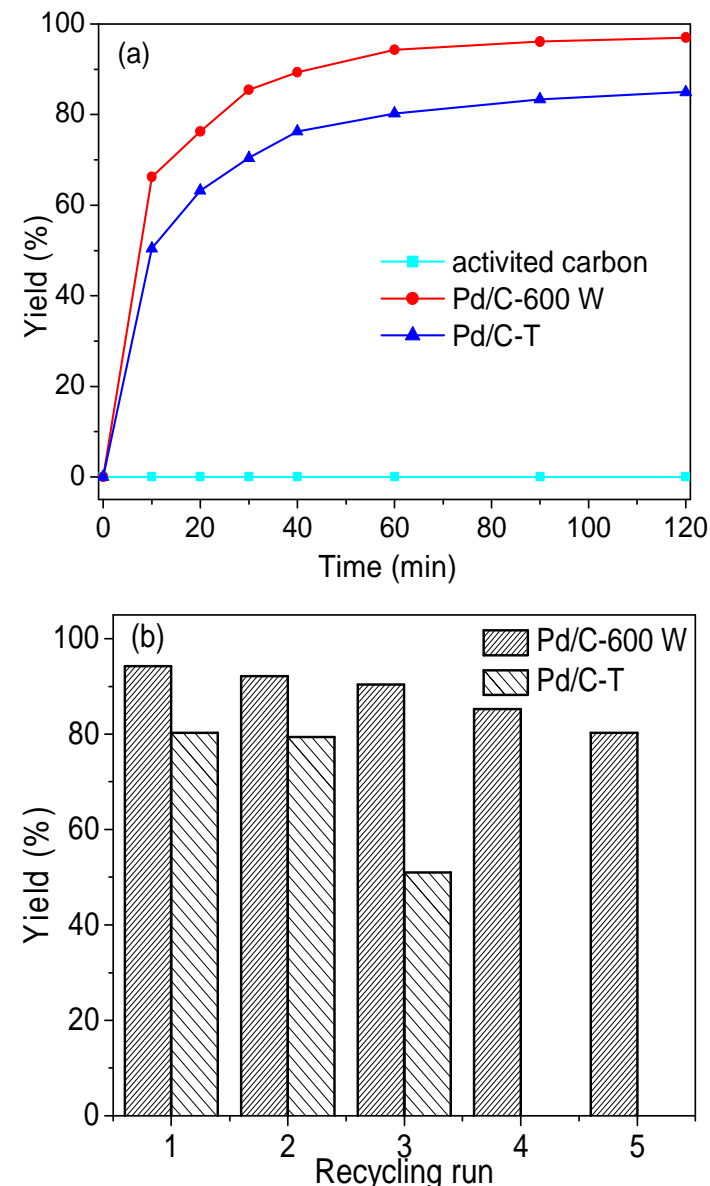

Fig. 5. (a) Yield of 4-bromotoluene at different intervals with various catalyst and (b) The recyclability of the Pd/C catalysts for the Suzuki-Miyaura coupling reaction

\section{SUMMARY}

In conclusion, we have succeeded in synthetizing $\mathrm{Pd} / \mathrm{C}-600 \mathrm{~W}$ catalyst for green Suzuki-Miyaura coupling reaction by facile one-pot ultrasonic reduction. The Pd NPs with an average particle size of $7.54 \mathrm{~nm}$ were uniformly dispersed on the active carbon surface in Pd/C-600W catalyst without any protective agent. The catalyst shows high activity toward Suzuki-Miyaura coupling reaction under mild conditions employed only $0.1 \mathrm{mmol} \%$ amount of catalyst. In addition, the yield of $\mathrm{d} / \mathrm{C}-600 \mathrm{~W}$ catalyst retained $80 \%$ after recycling five times.

\section{REFERENCES}

[1] K. Matos and J.A. Soderquist, "Alkylboranes in the Suzuki-Miyaura Coupling: Stereochemical and Mechanistic Studies". J. Organ. Chem. vol. 63, pp. 461-470, 1998.

[2] N. Razavi, B. Akhlaghinia and R. Jahanshahi, "Aminophosphine Palladium, pp. 0 Complex Supported on $\mathrm{ZrO} 2$ Nanoparticles, pp. ZrO$2 @ A E P H 2-P P h 2-P d(0$ as an Efficient Heterogeneous Catalyst for Suzuki-Miyaura and Heck-Mizoroki Reactions in Green Media". Catal. Lett. vol. 147, pp. 360-373, 2017.

[3] T. Bortolotto, S.E. Facchinetto, S.G. Trindade, A. Ossig, C.L. Petzhold, J. Vargas, O.E.D. Rodrigues, C. Giacomelli and V. Schmidt, "Polymer-coated palladium nanoparticle catalysts for Suzuki coupling reactions". J. Coll. Interf. Sci. vol. 439, pp. 154-161, 2015.

[4] X. Liu, L. Duan, J. Mo, E. Du, J. Shen, X. Lu, Y. Zhang, X. Zhou, C. He and F. Zhang, "Nitrogen deposition and its ecological impact in China: An overview". Envir. Pollut. vol. 159, pp. 2251-2264, 2011.

[5] H. Xu, B.W. Zeiger and K.S. Suslick, "Sonochemical synthesis of nanomaterials". Chem. Soc. Rev. vol. 42, pp. 2555-2267, 2013.

[6] D. Bhuyan, K. Selvaraj and L. Saikia, "Pd@SBA-15 nanocomposite catalyst: Synthesis and efficient solvent-free semihydrogenation of phenylacetylene under mild conditions". Micropor. Mesopor. Mater. vol. 241, pp. 266-273, 2017.

[7] Y. Gong, P. Zhang, X. Xu, Y. Li, H. Li and Y. Wang, "A novel catalyst Pd@ompg-C3N4 for highly chemoselective hydrogenation of quinoline under mild conditions". J. Catal. vol. 297, pp. 272-280, 2013.

[8] J. Li and X. Bai, "Ultrasonic synthesis of supported palladium nanoparticles for room-temperature Suzuki-Miyaura coupling". J. Mater. Sci. vol. 51, pp. 9108-9122, 2016. 